\title{
Clinicopathological Characteristics and Outcomes of Chinese Patients with Scanty Immune Deposits Lupus Nephritis: A Large Cohort Study from a Single Center
}

\author{
Qiuyu Li, ${ }^{1,2,3,4}$ Di Song, ${ }^{1,2,3,4}$ Fengmei Wang, ${ }^{1,2,3,4}$ Ying Tan, ${ }^{1,2,3,4}$ \\ Feng Yu, ${ }^{1,2,3,4}$ and Minghui Zhao ${ }^{1,2,3,4}$ \\ ${ }^{1}$ Renal Division, Department of Medicine, Peking University First Hospital, Beijing 100034, China \\ ${ }^{2}$ Institute of Nephrology, Peking University, Beijing 100034, China \\ ${ }^{3}$ Key Laboratory of Renal Disease, Ministry of Health of China, Beijing 100034, China \\ ${ }^{4}$ Key Laboratory of Chronic Kidney Disease Prevention and Treatment, Ministry of Education, Beijing 100034, China \\ Correspondence should be addressed to Feng Yu; yufengevert1@sina.com
}

Received 10 August 2013; Accepted 31 October 2013; Published 4 February 2014

Academic Editors: T. Gohda, Y. Iwata, and R. Liu

Copyright (C) 2014 Qiuyu Li et al. This is an open access article distributed under the Creative Commons Attribution License, which permits unrestricted use, distribution, and reproduction in any medium, provided the original work is properly cited.

\begin{abstract}
Objective. To assess clinicopathological characteristics of lupus nephritis patients with scanty immune deposits. Methods. The data of patients with scanty immune deposits lupus nephritis were retrospectively analyzed. Plasma ANCA and complement components were detected. Results. Among 316 cases with renal biopsy-proven lupus nephritis, 40 cases were diagnosed as scanty immune deposits. There were significantly higher value of serum creatinine $(P=0.002)$ and lower hemoglobin level $(P=0.009)$ and higher score of cellular crescents $(P=0.015)$ in scanty immune deposits group compared with immune complex deposits group. The frequency of positive plasma ANCA was significantly higher in scanty immune deposits group than that in immune complex deposits group (52.5\% versus $10.1 \%, P<0.001)$. As for comparisons of plasma complement components, there were significantly higher levels of $\mathrm{Clq}(P=0.005)$ and $\mathrm{Bb}(P=0.02)$ and lower level of factor $\mathrm{H}(P=0.003)$ in scanty immune deposits group. The ratio of treatment failure was significantly higher in scanty immune deposits group than that in immune deposits group (42.5\% versus $19.20 \%, P=0.001)$. The renal outcomes were similar between the two groups. Conclusions. Patients with scanty immune deposits lupus nephritis had more severe kidney damage. ANCA and activation of complement alternative pathway might be involved in the pathogenesis of the disease.
\end{abstract}

\section{Introduction}

Systemic lupus erythematosus (SLE) is a prototypic autoimmune disease characterized by the production of multiple autoantibodies. Renal involvement is common in SLE. The typical feature in lupus nephritis is immune complex deposition, showed as "full house" under immunofluorescence observation. However, in previous reports, some patients with lupus nephritis presented with "scanty immune deposits," that is, nonclassical glomerulonephritis, which might contribute to thrombotic microangiopathy (TMA) [1], ANCA-associated crescentic glomerulonephritis [2] podocytopathy [3], and so forth. Here, the "scanty immune deposits" were indicated as a descriptive term to identify the specimens with little or no staining for immunoglobulin and not nece- ssarily for lesions with necrosis or crescents. The clinicopathological features, outcomes, and possible pathogenesis of scanty immune deposits lupus nephritis have not been well delineated and extensively studied.

This study is to assess clinical manifestations, laboratory characteristics, pathological features, and outcomes of patients with scanty immune deposits in a large cohort of Chinese lupus nephritis patients. Particularly, we further detect the distribution of ANCA and complement activation profile in the patients.

\section{Methods}

2.1. Patients. Renal histopathological data of 316 patients with renal biopsy-proven lupus nephritis, diagnosed between 
January 2000 and July 2008 in Peking University First Hospital, were reviewed and reclassified according to the International Society of Nephrology and Renal Pathology Society (ISN/RPS) 2003 classification [4]. Only biopsy specimens with more than 10 glomeruli were included in the study.

On frozen sections of renal biopsy, at least two glomeruli, except for the sclerosed glomeruli, were evaluated by a renal pathologist. Scanty immune deposition was defined as negative staining or $1+$ positivity (on a scale of $0-4+$ ) of immunoglobulins (IgG, $\operatorname{IgA}$, and $\operatorname{IgM}$ ) by direct immunofluorescence assay and no electron-dense deposit in glomeruli, tubular basement membrane, and vessels was observed by electron microscopy assay. Immune complex deposits were defined as (i) a score of $2+$ or higher in staining for any kind of immunoglobulin observed by immunofluorescence microscopy and (ii) electron-dense deposits observed by electron microscopy [5].

The patients fulfilled the 1997 American College of Rheumatology revised criteria for SLE [6].

2.2. Clinical Evaluation. The following clinical data were collected and analyzed: gender, fever, malar rash, photosensitivity, oral ulcer, alopecia, arthritis, serositis, neurologic disorder, anemia, leukocytopenia, thrombocytopenia, hematuria, and leukocyturia. The criteria for system involvement were consistent with the 1997 American College of Rheumatology revised criteria for SLE [6]. The clinical disease activity was assessed by the Systemic Lupus Erythematosus Disease Activity Index (SLEDAI) [7, 8].

The renal response to the therapy includes complete remission, partial remission and treatment failure was detailed in previous studies [9-12].

A relapse was defined as (1) nephritic relapse: a recent increase of serum creatinine by $>50 \%$ with active urinary sediments; (2) proteinuric relapse: development of either a nephrotic syndrome (proteinuria $>3.5 \mathrm{~g} /$ day and serum albumin $<30 \mathrm{~g} / \mathrm{L}$ ) or proteinuria $>1.5 \mathrm{~g} /$ day without other causes, in previously nonproteinuric patients $[13,14]$.

The patients were followed up in outpatient clinic specified for patients with lupus nephritis. The primary end point was defined as death and the secondary end points were defined as end-stage renal disease (ESRD) or doubling of serum creatinine.

2.3. Laboratory Assessment. The following laboratory features were further detected using serum or plasma at the day of renal biopsy.

Serum antinuclear antibodies (ANA) were detected using indirect immunofluorescence assay (EUROIMMUN, Lübeck, Germany) and anti-double-stranded DNA (dsDNA) antibodies were detected using Crithidia luciliae indirect immunofluorescence test (EUROIMMUN, Lübeck, Germany). Anti-extractable nuclear antigen (ENA) antibodies, including anti-Sm, anti-SSA, anti-SSB, and anti-RNP antibodies, were detected using immunodotting assay (EUROIMMUN, Lübeck, Germany). Anti-cardiolipin antibodies and anti- $\beta_{2}$ GP-1 antibodies were detected using enzyme-linked immunosorbent assay (ELISA) (EUROIMMUN, Lübeck, Germany).
2.3.1. Detection of ANCA. ANCA tests were performed by both indirect immunofluorescence (IIF) assay and antigenspecific enzyme-linked immunosorbent assay (ELISA). Standard IIF assay was performed using precooled ethanol fixed normal peripheral neutrophils as substrate according to the manufacturer (EUROIMMUN, Lübeck, Germany). The use of Hep-2 cell and paraformaldehyde-fixed neutrophils may allow the distinction between ANA and p-ANCA. In antigenspecific ELISA, two highly purified known ANCA antigens, PR3 and MPO, purified as previously reported [15] were used as solid-phase ligands.

\subsubsection{Quantification of Plasma Complement Components.} Plasma concentrations of major human complement components were determined by enzyme-linked immunoassays, including complement fragments C5a (Quidel Corporation, San Diego, CA), C3a (Quidel Corporation, San Diego, CA), $\mathrm{Bb}$ (Quidel Corporation, San Diego, CA), soluble C5b-9 (SC5b-9, Quidel Corporation, San Diego, CA), properdin (Uscnk Life Science Inc., Wuhan, China), and C3 (Quidel Corporation, San Diego, CA). All the complement components were assayed according to the manufacturer's instructions. The principle of the assays was a four-step procedure: (I) microassay plates were precoated with murine monoclonal antibodies binding specifically to the complement components; (II) plasma samples were added according to the optimal dilutions, incubation time, and temperature from the instructions; (III) horseradish peroxidase conjugated antibodies binding to the complement components adsorbed on the plates were added; (IV) chromogenic substrate was added to determine the concentration of components.

The methods to detect plasma C4BP, Clq, and MBL (mannan-binding lectin) were the same as previously described with mild modification [16-18].

2.4. Renal Histopathology. The renal biopsy specimens were examined by light microscopy, direct immunofluorescence, and electron microscopy techniques.

2.4.1. Light Microscopy Examination. Renal biopsy specimens were fixed in $4.5 \%$ buffered formaldehyde for light microscopy. Consecutive serial $3 \mu \mathrm{m}$ sections were used for histological staining. Stains employed included haematoxylin and eosin (H\&E), periodic acid-Schiff (PAS), silver methenamine (Meth), and Masson's trichrome.

Crescentic glomerulonephritis was defined as over half of the total glomeruli affected by large crescents (the crescent takes up over half space in Bowman's capsule) by light microscope, which should be included in class IV-G lupus nephritis [5].

Renal thrombotic microangiopathy (TMA) was characterized by interlobular artery, arteriole, and glomerular capillary lesions, including endothelial cell swelling, lumen narrowing or obliteration, and thrombi formation by light microscopy. Swelling of glomerular endothelial cells, detachment from the glomerular basement membrane, and widening of the subendothelial space were identified by electron microscopy [19]. 

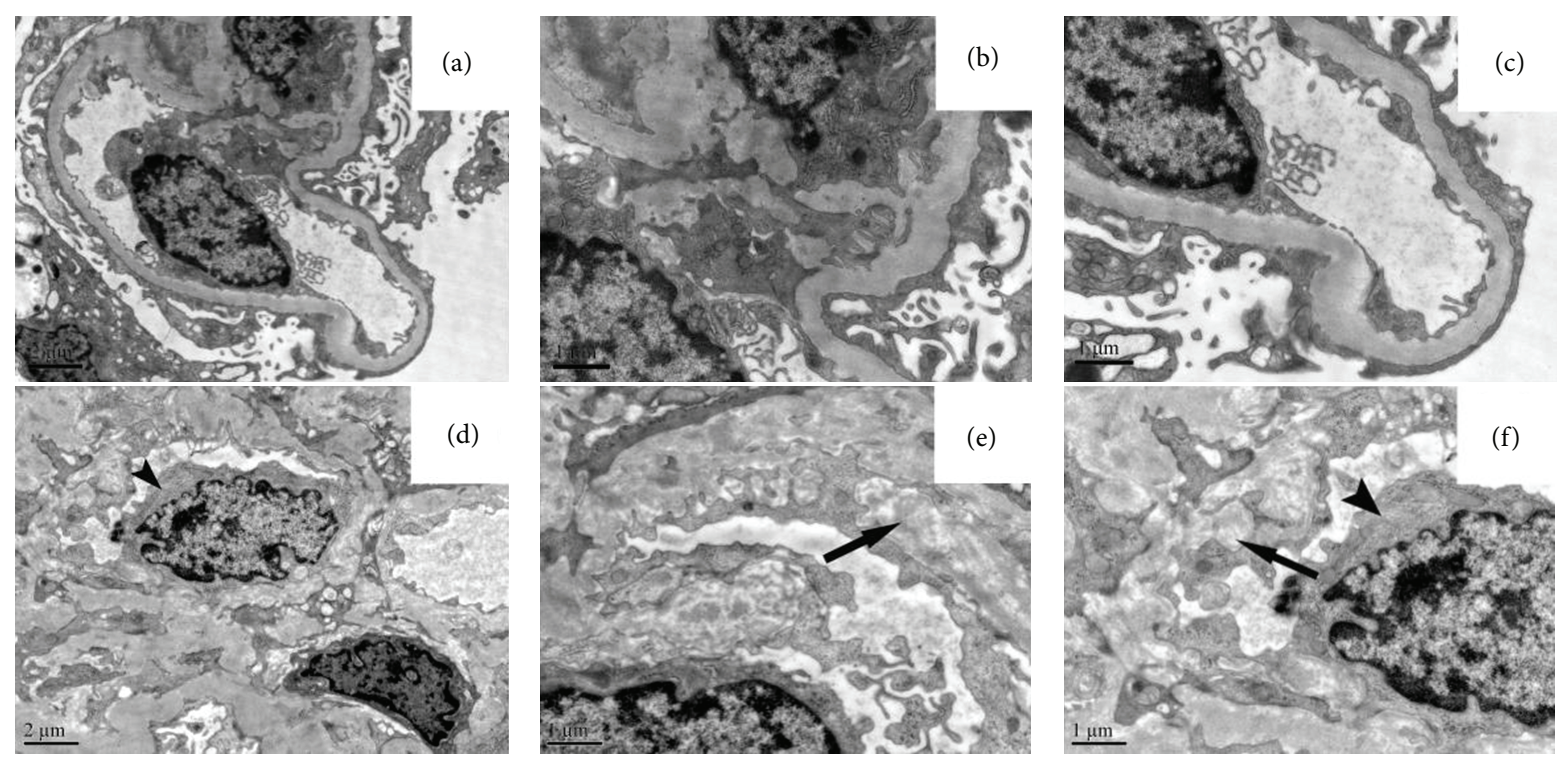

FIGURE 1: Electron micrographs of cases with scanty immune deposits lupus nephritis. (a)-(c) showed one case of mesangial proliferative lupus nephritis. No electron dense deposits were seen in mesangial area and glomerular basement membrane. Diffuse effacement of foot processes was observed. (d)-(f) showed one case of diffuse proliferative lupus nephritis combined with renal thrombotic microangiopathy. Glomerular endothelial cell (black pointer) was swollen, with increased mesangial matrix (d). Severe widening of subendothelial space (black arrow) with fluffy material and irregular cell projections; few of electron dense deposits were identified at higher magnification ((e), (f)). ((a), $($ d), original mag. $\times 10000)((b),(c),(e)$, and (f), original mag. $\times 20000)$.

Podocytopathy was defined as podocyte effacement. Biopsy findings revealed either no glomerular immune deposits or sparse deposits, which were confined to the glomerular mesangium. The characteristic pathological glomerular abnormality was ultrastructural and resided in the visceral glomerular epithelial cells. The glomerular lesions included idiopathic minimal change glomerulopathy and focal and segmental glomerulosclerosis $[3,20]$.

Pathological parameters such as activity indices (AI) and chronicity indices (CI) were approached by renal pathologists using a modification of a previously reported system involving semiquantitative scoring of specific biopsy features $[21,22]$.

2.4.2. Direct Immunofluorescence Examination. Direct immunofluorescence for immunoglobulin $\mathrm{G}$ (IgG), immunoglobulin A (IgA), immunoglobulin M (IgM), C3, Clq, and fibrin deposits was semiquantitatively graded from 0 to 4 according to the intensity of fluorescence. The glomeruli with sclerosis were excluded.

2.4.3. Electron Microscopy Examination. Renal biopsy specimens were fixed in $2.5 \%$ paraformaldehyde for electron microscopy. After being embedded in epon, ultrathin sections were mounted on metal grids and stained with uranyl acetate before being viewed in a transmission electron microscope (JEM-1230; JEOL, Tokyo, Japan).

2.5. Blood Samples. For detection of ANCA and complement, plasma samples were obtained from peripheral blood at the same day of renal biopsy before initiation of immunosuppressive treatment. The blood samples of patients and controls were drawn into EDTA tubes. The plasma was collected immediately by centrifugation at $2000 \mathrm{~g}$ for $15 \mathrm{~min}$ at $4^{\circ} \mathrm{C}$. All plasma samples were stored at $-80^{\circ} \mathrm{C}$ until use. Repeated freeze/thaw cycles were avoided.

Informed consent was obtained for blood sampling and renal biopsy from each patient. The research was in compliance of the Declaration of Helsinki. The design of this work was approved by the local ethical committees.

2.6. Statistical Analysis. Statistical software SPSS 16.0 (SPSS, Chicago, IL, USA) was employed for statistical analysis. Quantitative data were expressed as mean \pm SD, and median with range (minimum, maximum). For comparison of clinical and pathological features of patients, Student's $t$-test, oneway ANOVA analysis of variance, and Chi-square test were used. Kaplan-Meier curves were used to analyze patients' prognosis. Survival analysis was performed using the logrank test. Results were expressed as hazard ratio (HR) with $95 \%$ confidence intervals (CI). Statistical significance was considered as $P<0.05$.

\section{Results}

3.1. General Data of Patients with Scanty Immune Deposits Lupus Nephritis. Among the 316 lupus nephritis patients enrolled in the study, 40 cases $(12.66 \%)$ met the pathological criteria of scanty immune deposits nephritis, which were confirmed by electron microscopy (Figure 1).

In the scanty immune deposits group, 6 were male and 34 were female, with an average age of $39.78 \pm 12.90$ years at presentation. The majority of the patients (80\%) were with hematuria. Half of the patients were with leukocyturia and 
TABLE 1: Comparison of clinical data between patients with scanty immune deposits and immune complex deposits lupus nephritis.

\begin{tabular}{|c|c|c|c|}
\hline & Scanty immune deposits & Immune complex deposits & $P$ value \\
\hline Number of patients & 40 & 276 & \\
\hline Age $($ mean $\pm S D)$ (years) & $39.78 \pm 12.90$ & $32.06 \pm 10.94$ & $<0.001$ \\
\hline Gender (male/female) & $6 / 34$ & $42 / 234$ & 0.971 \\
\hline Number with fever (noninfection) (\%) & $11(27.5)$ & $82(29.7)$ & 0.774 \\
\hline Number with malar rash (\%) & $17(42.5)$ & $149(54.0)$ & 0.174 \\
\hline Number with photosensitivity (\%) & $6(15.0)$ & $57(20.7)$ & 0.403 \\
\hline Number with alopecia (\%) & $14(35.0)$ & $84(30.4)$ & 0.560 \\
\hline Number with oral ulcer (\%) & $11(23.9)$ & $83(30.1)$ & 0.739 \\
\hline Number with arthritis (\%) & $25(62.5)$ & $143(51.8)$ & 0.205 \\
\hline Number with serositis (\%) & $5(12.5)$ & $46(16.7)$ & 0.503 \\
\hline Number with neurologic disorder (\%) & $3(7.5)$ & $23(8.3)$ & 0.858 \\
\hline Number with anemia (\%) & $31(77.5)$ & $183(66.5)$ & 0.165 \\
\hline Number with leukocytopenia (\%) & $23(57.5)$ & $123(44.6)$ & 0.125 \\
\hline Number with thrombocytopenia (\%) & $10(25.0)$ & $93(33.8)$ & 0.267 \\
\hline Number with hematuria (\%) & $32(80.0)$ & $210(76.1)$ & 0.585 \\
\hline Number with leukocyturia (noninfection) (\%) & $23(57.5)$ & $145(52.5)$ & 0.557 \\
\hline Number with nephrotic syndrome (\%) & $25(62.5)$ & $161(58.8)$ & 0.653 \\
\hline SLEDAI (median; interquartile range) & $18,13-23$ & $17,14-21$ & 0.751 \\
\hline
\end{tabular}

$P<0.05$, italic values refer to the significant value between the two groups.

$62.5 \%$ with nephrotic syndrome. The median amount of urine protein was $4.04 \mathrm{~g} / 24$ hours. The median value of serum creatinine was $102 \mathrm{mmol} / \mathrm{dL}(80.25-189.50 \mathrm{mmol} / \mathrm{dL})$ upon diagnosis. The mean level of SLEDAI was $17.92 \pm 5.45$.

According to the 2003 classification of lupus nephritis, 6 patients were classified as class II (15\%, including 1 case combined with minimal change disease), 9 cases as class III (22.5\%), and 25 cases as class IV (62.5\%, including 1 case combined with TMA and 7 cases with crescentic glomerulonephritis).

All of the patients received oral prednisone therapy. The majority of patients completed treatment with oral cyclophosphamide (3/40) or monthly intravenous cyclophosphamide (600-800 mg/month) (20/40). The other patients received mycophenolate mofetil (3/40), leflunomide (8/40), and azathioprine (4/40). Two patients received prednisone alone. Twenty-six patients achieved clinical remission, 8 with complete remission and 18 with partial remission. Fourteen patients presented with treatment failure.

We further compared the clinical and pathological characteristics of scanty immune deposits and immune complex deposits patients with lupus nephritis.

3.2. Comparison of Clinical and Laboratory Parameters between Patients with Scanty Immune Deposits and Immune Complex Deposits Lupus Nephritis. The clinical and laboratory features of patients in the two groups were listed in Tables 1 and 2 .

The average age was significantly older in scanty immune deposits group than that in immune complex deposits group $(P<0.001)$. There were no significant differences between the two groups in other clinical indices.

In laboratory findings, there were significantly lower hemoglobin level $(P=0.009)$ and higher value of serum creatinine $(P=0.01)$ in scanty immune deposits group than those in immune complex deposits group.
Twenty-one out of the 40 patients (52.5\%) in scanty immune deposits group were ANCA positive including 15 with p-ANCA and 2 with c-ANCA by IIF, 10 with anti-MPO antibodies, and 1 with anti-PR3 antibodies by ELISA. 28 out of the 276 patients $(10.1 \%)$ in immune complex deposits group were ANCA positive including 20 with p-ANCA by IIF and 9 with anti-MPO by ELISA. The difference was significant $(P<0.001)$.

\subsection{Comparison of Plasma Complement Components Levels} between Patients with Scanty Immune Deposits And Immune Complex Deposits Lupus Nephritis. The levels of plasma complement components of patients in the two groups were listed in Table 3.

The normal levels of plasma MBL, C3a, C5a, and soluble C5b-9 were $1532 \pm 1020 \mathrm{ng} / \mathrm{mL}, 100.87 \pm 70.55 \mathrm{ng} / \mathrm{mL}, 9.32 \pm$ $7.88 \mathrm{ng} / \mathrm{mL}$, and $467.41 \pm 545.23 \mathrm{ng} / \mathrm{mL}$, respectively. They were significantly higher in patients with scanty immune deposits and immune complex deposits lupus nephritis than those in normal controls $(P<0.01, P<0.01 ; P<0.01$, $P<0.01 ; P<0.01, P<0.01 ; P<0.01, P<0.01$, resp. $)$.

The normal levels of plasma $\mathrm{Clq}$, properdin, $\mathrm{Bb}, \mathrm{C} 4 \mathrm{BP}$, factor $\mathrm{H}$, and C3 were $61.96 \pm 10.50 \mu \mathrm{g} / \mathrm{mL}, 22.58 \pm$ $9.67 \mu \mathrm{g} / \mathrm{mL}, 0.69 \pm 0.45 \mu \mathrm{g} / \mathrm{mL}, 326.59 \pm 87.25 \mu \mathrm{g} / \mathrm{mL}, 515.04$ $\pm 134.08 \mu \mathrm{g} / \mathrm{mL}$, and $0.80 \pm 0.17 \mathrm{mg} / \mathrm{mL}$, respectively. They were significantly lower in patients with scanty immune deposits and immune complex deposits lupus nephritis than those in normal controls $(P<0.01, P<0.01 ; P<0.01$, $P<0.01 ; P<0.01, P<0.01 ; P<0.01, P<0.01 ; P<0.01$, $P<0.01 ; P<0.01, P<0.01$, resp.).

3.3.1. Plasma Levels of C1q. Clq is the first component in the classical pathway of complement activation. The level of Clq was significantly higher in patients with scanty immune deposits lupus nephritis than that in immune complex deposits lupus nephritis $(34.78 \pm 5.65 \mu \mathrm{g} / \mathrm{mL}$ versus $22.17 \pm$ $3.08 \mu \mathrm{g} / \mathrm{mL}, P=0.005)$. 
TABLE 2: Comparison of laboratory data between patients with scanty immune deposits and immune complex deposits lupus nephritis.

\begin{tabular}{|c|c|c|c|}
\hline & $\begin{array}{c}\text { Scanty immune } \\
\text { deposits }\end{array}$ & $\begin{array}{l}\text { Immune complex } \\
\text { deposits }\end{array}$ & $P$ value \\
\hline Number of patients & 40 & 276 & \\
\hline Hemoglobin $(g / L)($ mean \pm SD) & $91.18 \pm 24.39$ & $102.16 \pm 24.93$ & 0.009 \\
\hline $\begin{array}{l}\text { Urine protein (g/24 hours) } \\
\text { (median; interquartile range) }\end{array}$ & $\begin{array}{c}4.04 \\
1.96-5.36\end{array}$ & $\begin{array}{c}4.34 \\
2.20-7.07\end{array}$ & 0.348 \\
\hline $\begin{array}{l}\text { Serum creatinine (mmol/dL) } \\
\text { (median; interquartile range) }\end{array}$ & $\begin{array}{c}102, \\
80.25-189.50\end{array}$ & $\begin{array}{c}81 \\
67-126\end{array}$ & 0.01 \\
\hline Number with positive ANA (\%) & $39(97.5)$ & $273(98.9)$ & 1 \\
\hline Number with positive anti-dsDNA (\%) & $25(64.1)$ & $191(69.2)$ & 0.521 \\
\hline Number with positive anti-Sm (\%) & $13(33.3)$ & $69(25.0)$ & 0.267 \\
\hline Number with positive anti-SSA (\%) & $16(41.0)$ & $124(44.9)$ & 0.646 \\
\hline Number with positive anti-SSB (\%) & $3(7.9)$ & $32(11.6)$ & 0.493 \\
\hline Number with positive anti-RNP (\%) & $12(30.8)$ & $84(30.4)$ & 0.966 \\
\hline Number with positive anticardiolipin (\%) & $2(2 / 34,6.9 \%)$ & $17(17 / 223,7.6 \%)$ & 1 \\
\hline Number with positive anti- $\beta 2$ GP-1 (\%) & $4(4 / 34,6.9 \%)$ & $15(15 / 223,6.7 \%)$ & 1 \\
\hline Number with positive ANCA (\%) & $21(52.5)$ & $28(10.1)$ & $<0.001$ \\
\hline
\end{tabular}

$P<0.05$, italic values refer to the significant value between the two groups.

TABLE 3: Comparison of plasma complement components levels between patients with scanty immune deposits and immune complex deposits lupus nephritis.

\begin{tabular}{|c|c|c|c|c|}
\hline & $\begin{array}{c}\text { Scanty immune } \\
\text { deposits }\end{array}$ & $\begin{array}{c}\text { Immune complex } \\
\text { deposits }\end{array}$ & $P$ value & Normal range \\
\hline Number of patients & 40 & 276 & & \\
\hline $\mathrm{Clq}(\mathrm{ug} / \mathrm{mL})($ mean $\pm \mathrm{SD})$ & $34.78 \pm 5.65$ & $22.17 \pm 3.08$ & 0.005 & $61.96 \pm 10.50$ \\
\hline $\begin{array}{l}\text { MBL }(\mathrm{ng} / \mathrm{mL}) \\
\text { (median; interquartile range) }\end{array}$ & $\begin{array}{c}2223 \\
575-3719\end{array}$ & $\begin{array}{c}1768 \\
476-2879\end{array}$ & 0.663 & $1532 \pm 1020$ \\
\hline Properdin $(u g / m L)($ mean $\pm S D)$ & $13.26 \pm 6.32$ & $15.39 \pm 6.05$ & 0.379 & $22.58 \pm 9.67$ \\
\hline $\begin{array}{l}\mathrm{Bb}(\mathrm{ug} / \mathrm{mL}) \\
\text { (median; interquartile range) }\end{array}$ & $\begin{array}{c}1.81 \\
0.62-2.54\end{array}$ & $\begin{array}{c}1.01 \\
0.7-1.74\end{array}$ & 0.02 & $0.69 \pm 0.45$ \\
\hline $\mathrm{C} 4 \mathrm{BP}(\mathrm{ug} / \mathrm{mL})($ mean $\pm \mathrm{SD})$ & $243.23 \pm 131.54$ & $221.56 \pm 79.25$ & 0.262 & $326.59 \pm 87.25$ \\
\hline Factor $\mathrm{H}(\mathrm{ug} / \mathrm{mL})($ mean $\pm \mathrm{SD})$ & $302.19 \pm 110.47$ & $407.52 \pm 181.44$ & 0.002 & $515.04 \pm 134.08$ \\
\hline $\begin{array}{l}\mathrm{C} 3 \text { (mg/mL) } \\
\text { (median; interquartile range) }\end{array}$ & $\begin{array}{c}0.58 \\
0.38-1.22\end{array}$ & $\begin{array}{c}0.54 \\
0.31-0.81\end{array}$ & 0.671 & $0.80 \pm 0.17$ \\
\hline $\begin{array}{l}\mathrm{C} 3 \mathrm{a}(\mathrm{ng} / \mathrm{mL}) \\
\text { (median; interquartile range) }\end{array}$ & $\begin{array}{c}4.09 \\
1.5-801.27\end{array}$ & $\begin{array}{c}3.15 \\
1-562.32\end{array}$ & 0.321 & $100.87 \pm 70.55$ \\
\hline $\begin{array}{l}\text { C5a (ng/mL) } \\
\text { (median; interquartile range) }\end{array}$ & $\begin{array}{c}20.32 \\
11.26-30.79\end{array}$ & $\begin{array}{c}17.25 \\
10.41-29.33\end{array}$ & 0.454 & $9.32 \pm 7.88$ \\
\hline $\begin{array}{l}\text { Soluble C5b-9 (ng/mL) } \\
\text { (median; interquartile range) }\end{array}$ & $\begin{array}{l}3.00 \\
1-754.35\end{array}$ & $\begin{array}{c}4.10 \\
1-698.41\end{array}$ & 0.239 & $467.41 \pm 545.23$ \\
\hline
\end{tabular}

$P<0.05$, italic values refer to the significant value between the two groups.

3.3.2. Plasma Levels of MBL. MBL serves as a trigger for the activation of lectin pathway. There was no significant difference in MBL levels between patients with scanty immune deposits and immune complex deposits lupus nephritis $(P=$ $0.675)$.

3.3.3. Plasma Levels of C4BP. C4BP is the main fluid-phase inhibitor of the complement activation. It exerts inhibitory function by enhancing the decay of classical/lectin pathway $\mathrm{C} 3$ convertase, $\mathrm{C} 4 \mathrm{~b} 2 \mathrm{a}[23]$, as well as the alternative pathway C3 convertase, C3bBb [24].
There was no significant difference in C4BP levels between patients with scanty immune deposits and immune complex deposits lupus nephritis $(P=0.389)$.

3.3.4. Plasma Levels of Bb, Properdin, and Factor H. Properdin is critical in the stabilization of alternative pathway $\mathrm{C} 3$ convertase. $\mathrm{Bb}$ is an activation fragment of factor $\mathrm{B}$ in the alternative complement pathway. Complement factor $\mathrm{H}$ is an abundant plasma complement regulator that inhibits alternative pathway activation by inhibiting the formation and accelerating the decay of C3 convertase and acting 
as a complement factor I cofactor, which inactivates $\mathrm{C} 3 \mathrm{~b}$ to $\mathrm{iC} 3 \mathrm{~b}$. Measurement of properdin, $\mathrm{Bb}$, and factor $\mathrm{H}$ in plasma provided evidence for the activation of the alternative complement pathway [25-27].

The level of $\mathrm{Bb}$ was significantly higher in patients with scanty immune deposits lupus nephritis than that in immune complex deposits lupus nephritis (1.81; 0.62$2.54 \mu \mathrm{g} / \mathrm{mL}$ versus $1.01 ; 7-1.74 \mu \mathrm{g} / \mathrm{mL}, P=0.02)$. The level of factor $\mathrm{H}$ was significantly lower in patients with scanty immune deposits lupus nephritis than that in immune complex deposits lupus nephritis $(302.19 \pm 110.47 \mu \mathrm{g} / \mathrm{mL}$ versus $407.52 \pm 181.44 \mu \mathrm{g} / \mathrm{mL}, P=0.003)$. There was no significant difference in properdin level between patients with scanty immune deposits lupus nephritis and immune complex deposits lupus nephritis $(P=0.357)$.

3.3.5. Plasma Levels of C3, C3a, C5a, and SC5b-9. Activation of complement results in the conversion of $\mathrm{C} 3$ to $\mathrm{C} 3 \mathrm{a}$ and $\mathrm{C} 3 \mathrm{~b}$ and then the formation of a $\mathrm{C} 5$ convertase multimolecular enzyme capable of cleaving C5 to C5a and C5b. The terminal complement complex (C5b-9) is generated by the assembly of C5b through C9 as a consequence of activation of complement system. Therefore, we tested plasma C3, C3a, C5a, and soluble C5b-9 (SC5b-9) levels to reflect total complement activation in circulation.

There were no significant differences in plasma concentration of C3, C3a, C5a, and SC5b-9 between patients with scanty immune deposits and immune complex deposits lupus nephritis $(P=0.541, P=0.134, P=0.446$, and $P=0.227$, resp.).

3.4. Comparison of Renal Histopathologic Parameters between Patients with Scanty Immune Deposits and Immune Complex Deposits Lupus Nephritis. The renal histopathological features of patients with and without immune deposits lupus nephritis were listed in Table 4.

In comparison with immune deposits group, patients with scanty immune deposits group had significantly higher scores of cellular crescents $(P=0.015)$. There were no significant differences in other pathological indices between the two groups. The ratios of thrombotic microangiopathy, crescentic glomerulonephritis, and podocytopathy were not significantly different between the two groups $(2.17 \%$ versus $8.33 \%, P=0.242 ; 15.22 \%$ versus $9.78 \%, P=0.052 ; 2.17 \%$ versus $0, P=0.307$, resp.).

3.5. Comparison of Treatment and Outcomes between Patients with Scanty Immune Deposits and Immune Complex Deposits Lupus Nephritis. The treatment and outcomes of patients with and without immune deposits lupus nephritis were detailed in Table 5. There was no significant difference in treatment algorithm between the two groups. The rates of complete remission and partial remission were not significantly different. The incidence of treatment failure was significantly higher in scanty immune deposits group than that in immune complex deposits group (36.95\% versus $19.20 \%, P=0.007)$.

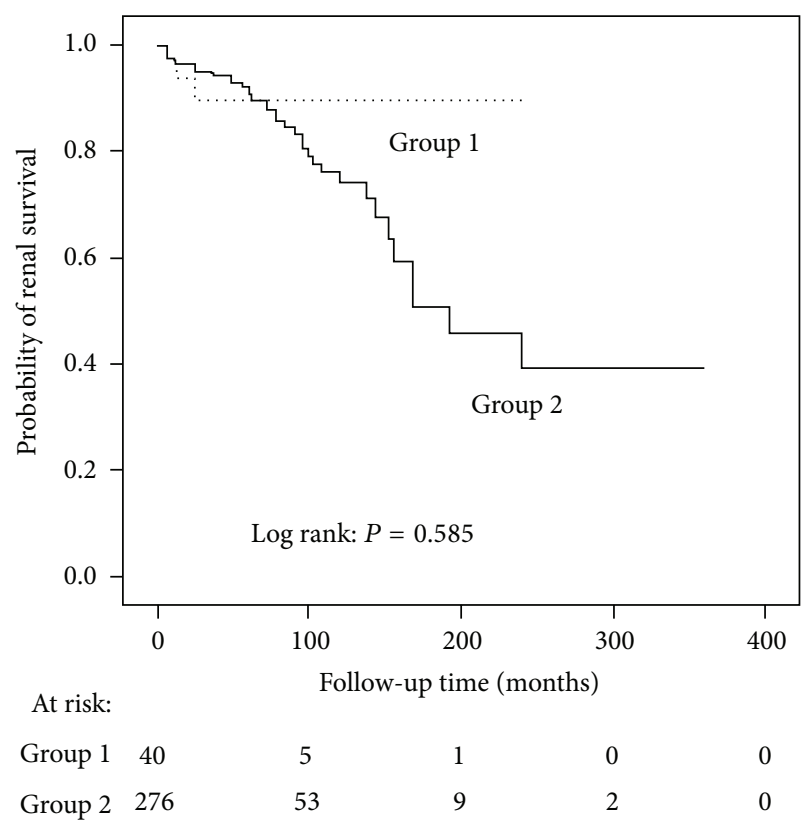

FIgURE 2: Comparison of renal outcomes between patients with scanty immune deposits (Group 1) and immune complex deposits lupus nephritis (Group 2).

During a similar follow-up time (average for nearly 5 years), the renal relapse rate was similar $(13.79 \%$ versus $12.56 \%, P=0.819$ ) between the two groups.

Regarding long-term survival, there were no significant differences in mortality and renal outcomes between scanty immune deposits and immune deposits groups $(P=0.598$, $P=0.585$, resp., Figure 2). In scanty immune deposits group, one patient died due to infection; 6 patients reached the secondary end point including 1 with doubling of serum creatinine and 5 with ESRD. In immune complex deposits group, 2 patients died due to heart failure and cerebral hemorrhage, respectively; 35 patients reached the secondary end point, all with ESRD.

We further compared renal outcomes between the scanty immune deposits class III lupus nephritis patients with those of immune complex deposits class III lupus nephritis and between scanty immune deposits crescentic lupus nephritis and immune complex deposits class IV lupus nephritis. In scanty immune deposits class III lupus nephritis group, 0 of the 9 patients reached the secondary end point. In immune complex deposits class III lupus nephritis group, one of the 40 patients reached the secondary end point. The ratio did not reach significant difference. In scanty immune deposits crescentic lupus nephritis group, 2 of the 7 patients reached the secondary end point. In immune complex deposits class IV lupus nephritis group, 30 of the 151 patients reached the secondary end point. Survival analysis showed that scanty immune deposits crescentic group had significantly worse renal outcome than that in immune complex deposits class IV group $(P=0.032$, Figure 3$)$.

Using the log-rank test for univariate survival analysis of renal prognosis in all the patients with lupus nephritis, we found that scanty immune deposits nephritis was not a risk 
TABLE 4: Comparison of renal pathological data between patients with scanty immune deposits and immune complex deposits lupus nephritis.

\begin{tabular}{|c|c|c|c|}
\hline & Scanty immune deposits & Immune complex deposits & $P$ value \\
\hline Number of biopsies & 40 & 276 & \multirow{6}{*}{0.195} \\
\hline Class II (\%) & $6(15)$ & $13(4.71)$ & \\
\hline Class III (\%) & $9(22.5)$ & $46(16.67)$ & \\
\hline Class IV (\%) & $25(62.5)$ & $151(54.7)$ & \\
\hline Class V (\%) & $0(0)$ & $64(23.2)$ & \\
\hline Class VI (\%) & $0(0)$ & $2(0.72)$ & \\
\hline $\begin{array}{l}\text { AI score } \\
(\text { mean } \pm \mathrm{SD})\end{array}$ & $8.70 \pm 4.58$ & $7.50 \pm 4.65$ & 0.128 \\
\hline $\begin{array}{l}\text { Endocapillary hypercellularity } \\
\text { (median; interquartile range) }\end{array}$ & $3,1-3$ & $3,1-3$ & 0.482 \\
\hline $\begin{array}{l}\text { Cellular crescents } \\
\text { (median; interquartile range) }\end{array}$ & $2,0-4$ & $0,0-2$ & 0.015 \\
\hline $\begin{array}{l}\text { Karyorrhexis/fibrinoid necrosis } \\
\text { (median; interquartile range) }\end{array}$ & $0,0-2$ & $0,0-2$ & 0.687 \\
\hline $\begin{array}{l}\text { Subendothelial hyaline deposits } \\
\text { (median; interquartile range) }\end{array}$ & $1,0-2$ & $1,0-2$ & 0.913 \\
\hline $\begin{array}{l}\text { Interstitial inflammation } \\
\text { (median; interquartile range) }\end{array}$ & $1,1-2$ & $1.1-1$ & 0.067 \\
\hline $\begin{array}{l}\text { Glomerular leukocyte infiltration } \\
\text { (median; interquartile range) }\end{array}$ & $1,0-1$ & $1,0-1$ & 0.694 \\
\hline $\begin{array}{l}\text { CI score } \\
(\text { mean } \pm \mathrm{SD})\end{array}$ & $3.10 \pm 2.29$ & $2.75 \pm 1.99$ & 0.31 \\
\hline $\begin{array}{l}\text { Glomerular sclerosis } \\
\text { (median; interquartile range) }\end{array}$ & $0,0-1$ & $0,0-1$ & 0.717 \\
\hline $\begin{array}{l}\text { Fibrous crescents } \\
\text { (median; interquartile range) }\end{array}$ & $0.0-0$ & $0,0-0$ & 0.795 \\
\hline $\begin{array}{l}\text { Tubular atrophy } \\
\text { (median; interquartile range) }\end{array}$ & $1,1-2$ & $1,1-1$ & 0.09 \\
\hline $\begin{array}{l}\text { Interstitial fibrosis } \\
\text { (median: interquartile range) }\end{array}$ & $1,1-1.75$ & $1,1-1$ & 0.182 \\
\hline
\end{tabular}

$P<0.05$, italic values refer to the significant value between the two groups.

factor for renal outcome in lupus nephritis. Other univariate risk factors included serum creatinine value, hemoglobin value, anti-SSB antibody, total activity indices score, cellular crescents, interstitial inflammatory cell infiltration, total chronicity indices score, fibrous crescents, and interstitial fibrosis (see Table 6 for details).

\section{Discussion}

It is well documented that various immunoglobulins deposited together with complements were found in the affected glomeruli in lupus nephritis. Accordingly, lupus nephritis was considered to be the classical type of immune complex associated glomerulonephritis. It is rare for lupus nephritis to contain no immune complex deposits, although there have been several cases of scanty immune deposits lupus nephritis reported previously [1-3, 28-30]. The clinicopathological features, outcomes, and possible pathogenesis of scanty immune deposits lupus nephritis should be studied.

The data arising from our study showed that the scanty immune deposits lupus nephritis was not uncommon, which accounted for $13 \%$ of all the lupus nephritis in our center. Of course, the glomeruli under pathological detection with sclerosis were excluded, and all the patients did not accept immunosuppressive treatment when the renal biopsies were done, which excluded the influences of therapy on the immune complex deposits in kidneys in our study. Previous studies indicated that the most possible explanation of scanty immune deposits lupus nephritis was that it might overlap with other scanty immune deposits-immune diseases, such as TMA [1], true renal vasculitis [29], ANCA-associated necrotizing and crescentic glomerulonephritis [2], glomerular podocytopathy [3], and glomerular lesions in kidney transplants [28]. Thus, we firstly focus on the presences of above disease in the scanty immune deposits group. We found that there were one patient with minimal change disease, one patient with TMA, and seven patients with true crescentic glomerulonephritis based on the strict diagnostic criteria $[3,5,9]$. But there was no significant difference in the ratios of the three pathological changes between scanty immune deposits group and immune complex deposits group.

After further comparisons of clinical, laboratory, and pathological features between the two groups, we found that 
TABLE 5: Comparison of treatment between patients with scanty immune deposits and immune complex deposits lupus nephritis.

\begin{tabular}{|c|c|c|c|}
\hline & Scanty immune deposits & Immune complex deposits & $P$ value \\
\hline Number of patients (\%) & 40 & 276 & \\
\hline \multicolumn{4}{|l|}{ Treatment } \\
\hline $\mathrm{P}$ & $40(100)$ & $276(100)$ & 1 \\
\hline CYC & $23(57.50)$ & $156(56.52)$ & 0.907 \\
\hline AZA & $4(10.00)$ & $21(7.60)$ & 0.833 \\
\hline MMF & $3(7.50)$ & $17(6.15)$ & 1 \\
\hline LEF & $8(20.00)$ & $31(11.23)$ & 0.187 \\
\hline \multicolumn{4}{|l|}{ Treatment response } \\
\hline CR & $8(20.00)$ & $78(28.26)$ & 0.560 \\
\hline PR & $18(45.00)$ & $145(52.54)$ & 0.373 \\
\hline $\mathrm{TF}$ & $14(35)$ & $53(19.20)$ & 0.001 \\
\hline Duration of followup (months) & $38,6-78$ & $48,8.5-84$ & 0.952 \\
\hline Relapse rate & $\begin{array}{c}4(4 / 26,15.38 \%, 3 \text { with } \\
\text { nephritic relapse and } 1 \text { with } \\
\text { proteinuric relapse) }\end{array}$ & $\begin{array}{c}28(28 / 223,12.56 \%, 20 \text { with } \\
\text { nephritic relapse and } 8 \text { with } \\
\text { proteinuric relapse })\end{array}$ & 0.922 \\
\hline
\end{tabular}

Note: P: oral prednisone; CYC: cyclophosphamide; AZA: azathioprine; MMF: mycophenolate mofetil; LEF: leflunomide; CR: complete remission; PR: partial remission; TF: treatment failure.

$P<0.05$, italic values refer to the significant value between the two groups.

TABLE 6: Univariate survival analysis of patients renal prognosis with lupus nephritis.

\begin{tabular}{|c|c|c|c|c|}
\hline & HR & \multicolumn{2}{|c|}{ 95\% confidence interval } & $P$ value \\
\hline Age & 0.240 & 0.056 & 1.031 & 0.055 \\
\hline Sex & 0.514 & 0.158 & 1.674 & 0.269 \\
\hline $\mathrm{C} 3$ & 0.693 & 0.167 & 2.876 & 0.613 \\
\hline Proteinuria & 0.948 & 0.226 & 3.971 & 0.942 \\
\hline Serum creatinine value & 16.063 & 6.746 & 38.251 & $<0.001$ \\
\hline Hemoglobin & 0.285 & 0.154 & 0.530 & $<0.001$ \\
\hline ANA & 0.389 & 0.053 & 2.855 & 0.353 \\
\hline Anti-ds-DNA antibody & 1.376 & 0.710 & 2.670 & 0.345 \\
\hline Anti-Sm antibody & 0.972 & 0.476 & 1.984 & 0.938 \\
\hline Anti-SSA antibody & 0.786 & 0.417 & 1.479 & 0.455 \\
\hline Anti-SSB antibody & 2.878 & 1.365 & 6.067 & 0.005 \\
\hline Anti-RNP antibody & 0.721 & 0.361 & 1.441 & 0.355 \\
\hline Anticardiolipin antibody & 1.175 & 0.350 & 3.939 & 0.794 \\
\hline SLEDAI & 0.933 & 0.255 & 4.430 & 0.933 \\
\hline Activity indices (AI) score & 3.941 & 1.876 & 8.279 & $<0.001$ \\
\hline Endocapillary hypercellularity & 1.814 & 0.803 & 4.099 & 0.152 \\
\hline Cellular crescents & 3.339 & 1.703 & 6.547 & $<0.001$ \\
\hline Karyorrhexis/fibrinoid necrosis & 1.646 & 0.890 & 3.043 & 0.112 \\
\hline Subendothelial hyaline deposits & 0.848 & 0.445 & 1.616 & 0.721 \\
\hline Interstitial inflammatory cell infiltration & 5.492 & 2.859 & 10.547 & $<0.001$ \\
\hline Glomerular leukocyte infiltration & 1.145 & 0.613 & 2.140 & 0.671 \\
\hline Chronicity indices (CIs) score & 1.379 & 1.230 & 1.545 & $<0.001$ \\
\hline Glomerular sclerosis & 1.025 & 0.243 & 4.320 & 0.793 \\
\hline Fibrous crescents & 3.412 & 1.839 & 6.328 & $<0.001$ \\
\hline Tubular atrophy & 25.129 & 0.497 & 1.271 & 0.107 \\
\hline Interstitial fibrosis & 9.222 & 1.268 & 67.070 & 0.028 \\
\hline Scanty immune deposits or immune complex deposits & 1.320 & 0.519 & 3.359 & 0.560 \\
\hline
\end{tabular}

$P<0.05$, italic values refer to the significant value between the two groups.

Bold values refers to that scanty immune deposits nephritis was not a risk factor for a renal outcome in lupus nephritis. 


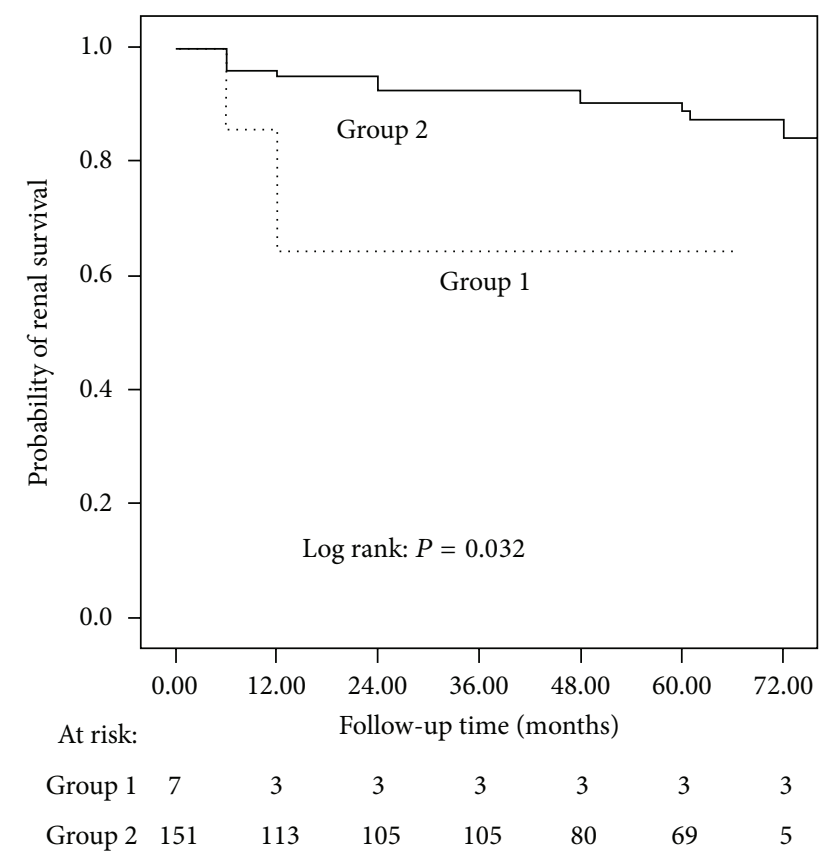

FIGURE 3: Comparison of renal outcomes between patients with scanty immune deposits crescentic lupus nephritis (Group 1) and immune complex deposits class IV lupus nephritis (Group 2).

the patients with scanty immune deposits lupus nephritis presented with older age, higher value of serum creatinine, and lower value of hemoglobin. Furthermore, significantly higher score of cellular crescents was found in scanty immune deposits group compared with immune complex deposits group evaluated by NIH scoring system. More importantly, by immunofluorescence assay and ELISA, we found that the positive ratio of ANCA was significantly higher in scanty immune deposits group than that in immune complex deposits group. Our previous study also showed that crescentic lupus nephritis presented with lower intensity of immunoglobulins and higher ratio of ANCA [31]. Thus, it highlights the importance of ANCA in the pathogenesis of scanty immune deposits crescentic lupus nephritis.

ANCA, as one of autoantibodies in SLE, might be implicated as a pathogenic factor for the development of scanty immune deposits crescentic lupus nephritis. Nasr et al. recently proposed that one of the two conditions (ANCA and lupus nephritis) may be creating fertile conditions for the second to develop [32]. It was suggested that lupus nephritis might facilitate the process of MPO autoantibody formation by promoting neutrophil degranulation and priming neutrophils to increase surface expression of MPO. Besides, the presence of ANCA was most closely associated with vasculitic lesions and the typical characteristic of ANCA-associated renal vasculitis was scanty immune deposits. So, it was suggested that the presence of ANCA in patients with SLE might indicate overlaps of SLE and ANCA-associated vasculitis [33], especially for those with the disproportionate necrotizing and crescentic features in lupus nephritis [31].

On the other hand, over half of the patients with scanty immune deposits lupus nephritis in our study were with
ANCA negative. Apart from ANCA, other mechanisms of developing scanty immune deposits lupus nephritis were thought of. The role of complement in SLE was important and it was closely associated with immune complex formation. So, we further detected the plasma complement components levels, including classical, alternative, and mannose-binding lectin (MBL) pathways in our patients with lupus nephritis. The results showed that there were significantly higher levels of $\mathrm{Clq}$ and $\mathrm{Bb}$ and lower level of factor $\mathrm{H}$ in scanty immune deposits group compared with immune complex deposits group, which indicated the predominant activation of the complement alternative pathway in the pathogenesis of scanty immune deposits lupus nephritis.

The role of complement activation played in SLE and lupus nephritis has been assumed for many years. It was acknowledged that immune complexes formed by selfantigens and autoantibodies might activate the classical pathway, generating inflammatory mediators and resulting in tissue damage. However, convincing evidences indicated that the alternative pathway was also activated and participated in the pathogenesis of SLE, especially in lupus nephritis [3439]. The analysis of complement components in our study showed that all the three pathways might be activated and involved in the pathogenesis of lupus nephritis. However, the higher levels of $\mathrm{Clq}$ and $\mathrm{Bb}$ indicated that alternative pathway activation, other than classical pathway, was more notable in scanty immune deposits lupus nephritis than that in immune complex deposits group. In addition, lower level of factor $\mathrm{H}$, an important inhibitory factor in alternative pathway by accelerating the decay of the alternative pathway $\mathrm{C} 3$-convertase $(\mathrm{C} 3 \mathrm{bBb})$, also supported that there might exist overactivation of alternative pathway. A recent study even showed that factor $\mathrm{H}$ deficiency could accelerate the development of lupus nephritis in lupus-prone mice MRL-lpr [40].

Interestingly, recent studies, including the mouse model of anti-MPO IgG mediated glomerulonephritis and human ANCA associated vasculitis, suggested that complement alternative pathway activation was crucial for ANCA associated vasculitis development [41-43]. Taken together, ANCA and complement alternative pathway activation might both be involved in the development of scanty immune deposits lupus nephritis which need further investigation.

There is no well-established guideline for the treatment of scanty immune deposits lupus nephritis. The therapy between scanty immune deposits and immune complex deposits groups in our patients was similar, both including immunosuppressive treatment. Although the ratio of renal remission rate was not different in the two groups, higher proportion of treatment failure was found in scanty immune deposits group. It might be related to the more severe clinical and pathological characteristics in scanty immune deposits group.

The 5-year survival rate and renal outcome between the two groups were similar. Scanty immune deposits nephritis was not a risk factor for renal outcome by log-rank test for univariate survival analysis of renal prognosis. However, as scanty immune deposits lupus nephritis consisted of a group of pathological types, we further compared renal outcomes between different subgroups. Interestingly, we found that 
scanty immune deposits crescentic group had significantly worse renal outcome than that in immune complex deposits class IV group, which needs further observation.

There were some limitations in this study: (I) despite being a large cohort study, it was a retrospective analysis; (II) the staining of complement components in kidney tissues, especially in alternative pathway, is important; (III) longer duration of followup and multicenter study are needed.

In conclusion, scanty immune deposits lupus nephritis was not uncommon. Patients with scanty immune deposits lupus nephritis had more severe kidney damage. ANCA-associated vasculitis and activation of complement alternative pathway might be involved in the pathogenesis of scanty immune deposits lupus nephritis.

\section{Conflict of Interests}

The authors declare that there is no conflict of interests regarding the publication of this paper.

\section{Authors' Contribution}

Qiu-yu Li and Di Song contributed equally to this work.

\section{Acknowledgments}

This work was supported by a Grant of Chinese 973 Project (no. 2012CB517702), National Natural Science Foundation of China to Innovation Research Group (no. 81021004), and National Natural Science Foundation of China (no. 81100497).

\section{References}

[1] D. A. Charney, G. Nassar, L. Truong, and T. Nadasdy, "Pauciimmune' proliferative and necrotizing glomerulonephritis with thrombotic microangiopathy in patients with systemic lupus erythematosus and lupus-like syndrome," The American Journal of Kidney Diseases, vol. 35, no. 6, pp. 1193-1206, 2000.

[2] A. Fayaz, Y. Pirson, J. P. Cosyns, J. Yango, and M. Lambert, "Pauci-immune necrotizing and crescentic glomerulonephritis in a patient with systemic lupus erythematosus," Clinical Nephrology, vol. 69, no. 4, pp. 290-293, 2008.

[3] S. W. Kraft, M. M. Schwartz, S. M. Korbet, and E. J. Lewis, "Glomerular podocytopathy in patients with systemic lupus erythematosus," Journal of the American Society of Nephrology, vol. 16, no. 1, pp. 175-179, 2005.

[4] J. J. Weening, V. D. D’Agati, M. M. Schwartz et al., "The classification of glomerulonephritis in systemic lupus ereythematosus revisited," Kidney International, vol. 65, pp. 521-530, 2004.

[5] R. J. Falk, J. C. Jennette, and P. H. Nachman, "Primary glomerular disease," in The Kidney, B. M. Brenner, Ed., pp. 1293-1380, Saunders, Philadelphia, Pa, USA, 7th edition, 2004.

[6] M. C. Hochberg, "Updating the American college of rheumatology revised criteria for the classification of systemic lupus erythematosus," Arthritis and Rheumatism, vol. 40, no. 9, article $1725,1997$.

[7] C. Bombardier, D. D. Gladman, M. B. Urowitz, D. Caron, and C. H. Chang, "Derivation of the SLEDAI: a disease activity index for lupus patients," Arthritis and Rheumatism, vol. 35, no. 6, pp. 630-640, 1992.

[8] M. H. Liang, S. A. Socher, M. G. Larson, and P. H. Schur, "Reliability and validity of six systems for the clinical assessment of disease activity in systemic lupus erythematosus," Arthritis and Rheumatism, vol. 32, no. 9, pp. 1107-1118, 1989.

[9] J. Wang, W. Hu, H. Xie et al., "Induction therapies for class IV lupus nephritis with non-inflammatory necrotizing vasculopathy: mycophenolate mofetil or intravenous cyclophosphamide," Lupus, vol. 16, no. 9, pp. 707-712, 2007.

[10] E. M. Ginzler, M. A. Dooley, C. Aranow et al., "Mycophenolate mofetil or intravenous cyclophosphamide for lupus nephritis," The New England Journal of Medicine, vol. 353, no. 21, pp. 22192228, 2005.

[11] T. M. Chan, K. C. Tse, C. S. O. Tang, K. N. Lai, and F. K. Li, "Long-term outcome of patients with diffuse proliferative lupus nephritis treated with prednisolone and oral cyclophosphamide followed by azathioprine," Lupus, vol. 14, no. 4, pp. 265-272, 2005.

[12] H. Y. Wang, T. G. Cui, F. F. Hou et al., "Induction treatment of proliferative lupus nephritis with leflunomide combined with prednisone: a prospective multi-centre observational study," Lupus, vol. 17, no. 7, pp. 638-644, 2008.

[13] G. Contreras, V. Pardo, B. Leclercq et al., "Sequential therapies for proliferative lupus nephritis," The New England Journal of Medicine, vol. 350, no. 10, pp. 971-980, 2004.

[14] C. Grootscholten, G. Ligtenberg, E. C. Hagen et al., "Azathioprine/methylprednisolone versus cyclophosphamide in proliferative lupus nephritis. A randomized controlled trial," Kidney International, vol. 70, no. 4, pp. 732-742, 2006.

[15] G. Xin, M. H. Zhao, and H. Y. Wang, "Detection rate and antigenic specificities of antineutrophil cytoplasmic antibodies in Chinese patients with clinically suspected vasculitis," Clinical and Diagnostic Laboratory Immunology, vol. 11, no. 3, pp. 559$562,2004$.

[16] A. F. Zadura, E. Theander, A. M. Blom, and L. A. Trouw, "Complement inhibitor C4b-binding protein in primary Sjögren's syndrome and its association with other disease markers," Scandinavian Journal of Immunology, vol. 69, no. 4, pp. 374-380, 2009.

[17] E. J. M. Nascimento, A. M. Silva, M. T. Cordeiro et al., "Alternative complement pathway deregulation is correlated with dengue severity," PLoS ONE, vol. 4, no. 8, Article ID e6782, 2009.

[18] E. Förster-Waldl, L. Cokoja, O. Förster, and W. Maurer, "Mannose-binding lectin: comparison of two assays for the quantification of MBL in the serum of pediatric patients," Journal of Immunological Methods, vol. 276, no. 1-2, pp. 143-146, 2003.

[19] G. B. Appel, C. L. Pirani, and V. D’Agati, "Renal vascular complications of systemic lupus erythematosus," Journal of the American Society of Nephrology, vol. 4, no. 8, pp. 1499-1515, 1994.

[20] G. S. Markowitz and V. D. D’Agati, "Classification of lupus nephritis," Current Opinion in Nephrology and Hypertension, vol. 18, no. 3, pp. 220-225, 2009.

[21] H. A. Austin III, D. T. Boumpas, E. M. Vaughan, and J. E. Balow, "Predicting renal outcomes in severe lupus nephritis: contributions of clinical and histologic data," Kidney International, vol. 45 , no. 2, pp. 544-550, 1994.

[22] H. A. Austin III, L. R. Muenz, K. M. Joyce, T. T. Antonovych, and J. E. Balow, "Diffuse proliferative lupus nephritis: identification of specific pathologic features affecting renal outcome," Kidney International, vol. 25, no. 4, pp. 689-695, 1984. 
[23] S. R. Barnum and B. Dahlback, "C4b-binding protein, a regulatory component of the classical pathway of complement, is an acute-phase protein and is elevated in systemic lupus erythematosus," Complement and Inflammation, vol. 7, no. 2, pp. 71-77, 1990.

[24] A. M. Blom, L. Kask, and B. Dahlbäck, "CCP1-4 of the C4b-binding protein $\alpha$-chain are required for factor I mediated cleavage of complement factor C3b," Molecular Immunology, vol. 39, no. 10, pp. 547-556, 2003.

[25] I. Y. Pavlov, N. de Forest, and J. C. Delgado, "Specificity of EIA immunoassay for complement factor Bb testing," Clinical Laboratory, vol. 57, no. 3-4, pp. 225-228, 2011.

[26] H. Watanabe, E. Noguchi, K. Shio, H. Iwadate, H. Kobayashi, and $\mathrm{H}$. Ohira, "Usefulness of complement split product, Bb, as a clinical marker for disease activity of lupus nephritis," Fukushima Journal of Medical Science, vol. 52, no. 2, pp. 103-109, 2006.

[27] M. T. Ganter, K. Brohi, M. J. Cohen et al., "Role of the alternative pathway in the early complement activation following major trauma," Shock, vol. 28, no. 1, pp. 29-34, 2007.

[28] S. M. Meehan, A. Chang, A. Khurana, R. Baligan, P. V. Kadambi, and B. Javaid, "Pauci-immune and immune glomerular lesions in kidney transplants for systemic lupus erythematosus," Clinical Journal of the American Society of Nephrology, vol. 3, no. 5, pp. 1469-1478, 2008.

[29] A. A. Abdellatif, S. Waris, A. Lakhani, H. Kadikoy, W. Haque, and L. D. Truong, "True vasculitis in lupus nephritis," Clinical Nephrology, vol. 74, no. 2, pp. 106-112, 2010.

[30] F. C. Li, D. Y. Hwang, C. C. Hung, and H. C. Chen, "Pauci-immune lupus nephritis: a case report," Kaohsiung Journal of Medical Sciences, vol. 24, no. 10, pp. 531-535, 2008.

[31] F. Yu, Y. Tan, G. Liu, S. X. Wang, W. Z. Zou, and M. H. Zhao, "Clinicopathological characteristics and outcomes of patients with crescentic lupus nephritis," Kidney International, vol. 76, no. 3, pp. 307-317, 2009.

[32] S. H. Nasr, V. D. D’Agati, H. R. Park et al., "Necrotizing and crescentic lupus nephritis with antineutrophil cytoplasmic antibody seropositivity," Clinical Journal of the American Society of Nephrology, vol. 3, no. 3, pp. 682-690, 2008.

[33] N. N. Masani, L. J. Imbriano, V. D. D’Agati, and G. S. Markowitz, "SLE and rapidly progressive glomerulonephritis," The American Journal of Kidney Diseases, vol. 45, no. 5, pp. 950-955, 2005.

[34] J. P. Buyon, J. Tamerius, S. Ordorica, B. Young, and S. B. Abramson, "Activation of the alternative complement pathway accompanies disease flares in systemic lupus erythematosus during pregnancy," Arthritis and Rheumatism, vol. 35, no. 1, pp. 55-61, 1992.

[35] J. P. Buyon, J. Tamerius, H. M. Belmont, and S. B. Abramson, "Assessment of disease activity and impending flare in patients with systemic lupus erythematosus: comparison of the use of complement split products and conventional measurements of complement," Arthritis and Rheumatism, vol. 35, no. 9, pp.10281037, 1992.

[36] L. D. Kerr, B. R. Adelsberg, P. Schulman, and H. Spiera, "Factor $\mathrm{B}$ activation products in patients with systemic lupus erythematosus. A marker of severe disease activity," Arthritis and Rheumatism, vol. 32, no. 11, pp. 1406-1413, 1989.

[37] N. Sato, I. Ohsawa, S. Nagamachi et al., "Significance of glomerular activation of the alternative pathway and lectin pathway in lupus nephritis," Lupus, vol. 20, no. 13, pp. 1378-1386, 2011.

[38] P. Sánchez-Corral, D. Bellavia, L. Amico, M. Brai, and S. R. de Córdoba, "Molecular basis or factor $\mathrm{H}$ and FHL-1 deficiency in an Italian family," Immunogenetics, vol. 51, no. 4-5, pp. 366-369, 2000.

[39] G. Nagy, M. Brózik, L. Varga et al., "Usefulness of detection of complement activation products in evaluating SLE activity," Lupus, vol. 9, no. 1, pp. 19-25, 2000.

[40] L. Bao, M. Haas, and R. J. Quigg, "Complement factor H deficiency accelerates development of lupus nephritis," Journal of the American Society of Nephrology, vol. 22, no. 2, pp. 285-295, 2011.

[41] J. C. Jennette and R. J. Falk, "New insight into the pathogenesis of vasculitis associated with antineutrophil cytoplasmic autoantibodies," Current Opinion in Rheumatology, vol. 20, no. 1, pp. 55-60, 2008.

[42] H. Xiao, A. Schreiber, P. Heeringa, R. J. Falk, and J. C. Jennette, "Alternative complement pathway in the pathogenesis of disease mediated by anti-neutrophil cytoplasmic autoantibodies," American Journal of Pathology, vol. 170, no. 1, pp. 52-64, 2007.

[43] G. Q. Xing, M. Chen, G. Liu, X. Zheng, E. J. Jie, and M. H. Zhao, "Differential deposition of C4d and MBL in glomeruli of patients with ANCA-negative pauci-immune crescentic glomerulonephritis," Journal of Clinical Immunology, vol. 30, no. 1, pp. 144-156, 2010. 


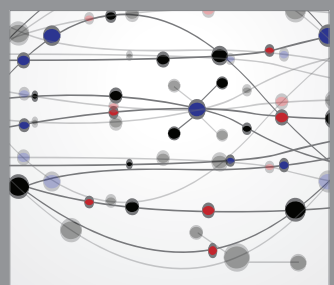

The Scientific World Journal
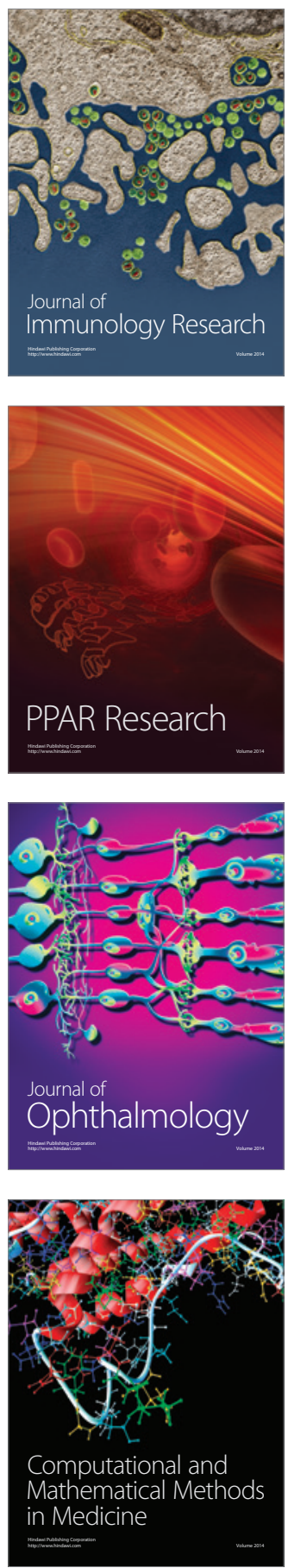

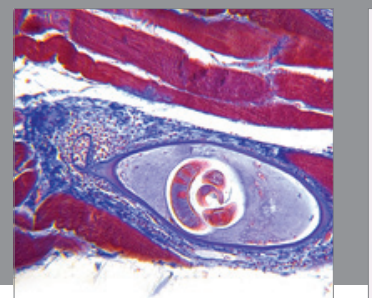

Gastroenterology

Research and Practice
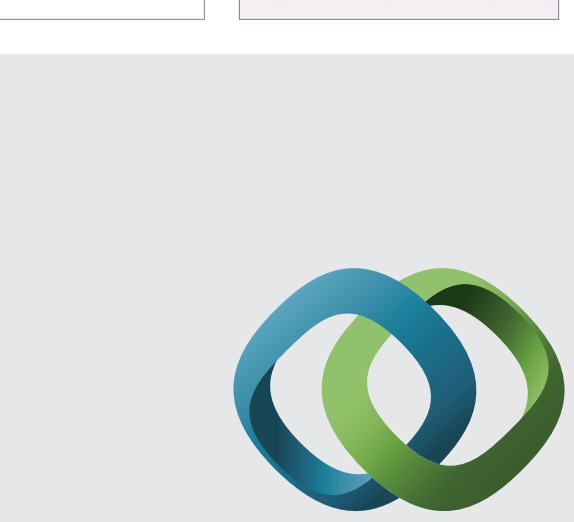

\section{Hindawi}

Submit your manuscripts at

http://www.hindawi.com
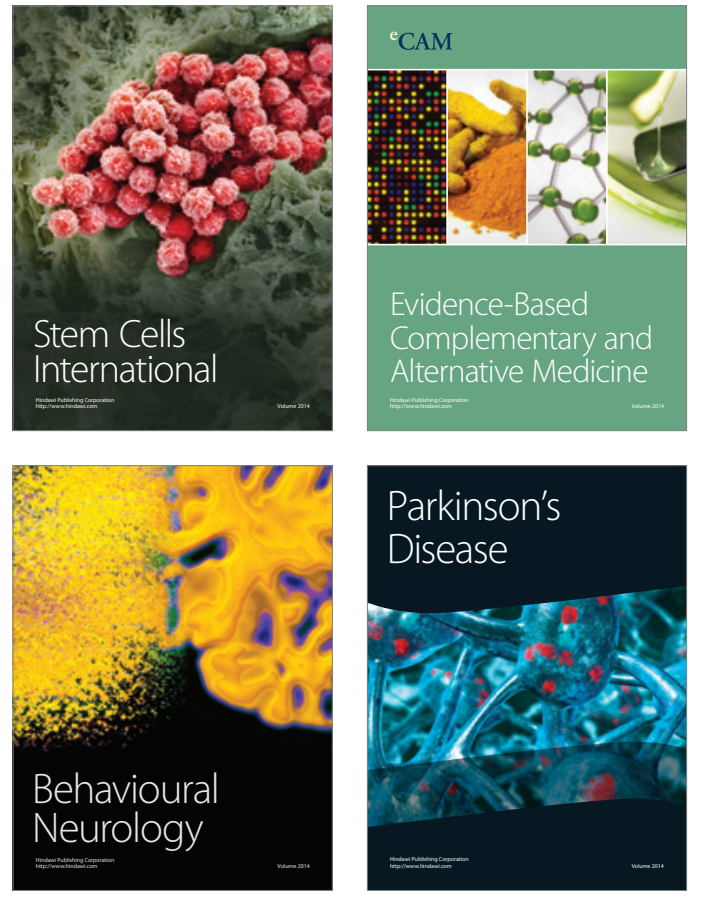
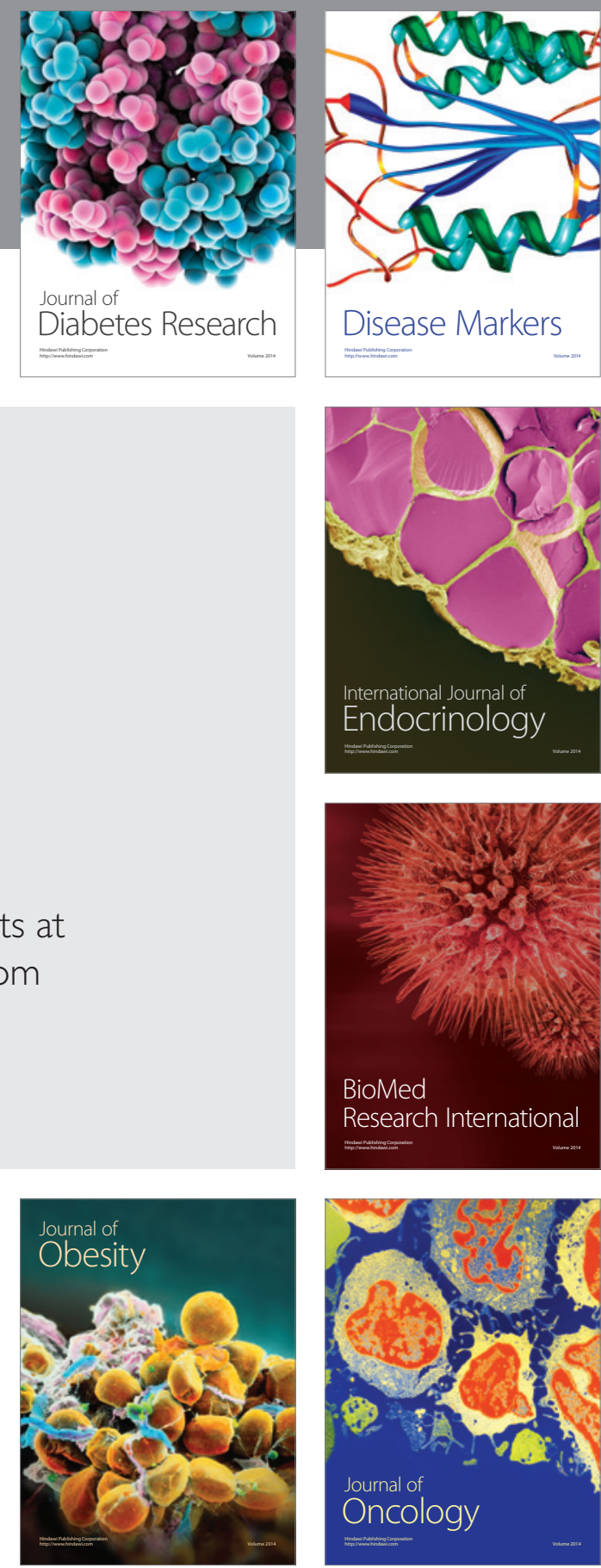

Disease Markers
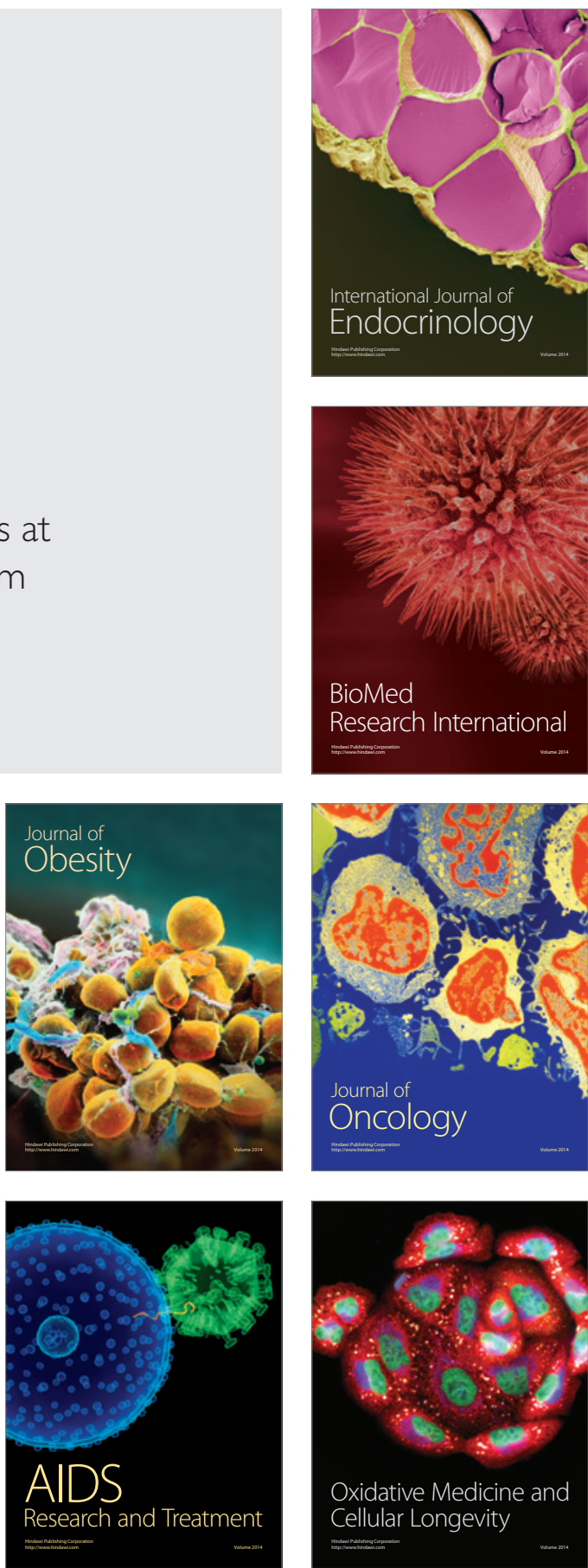